Fluid Dynamics Research

December 2014, Volume 46 Issue 6 Pages 061401

http://dx.doi.org/10.1088/0169-5983/46/6/061401

http://archimer.ifremer.fr/doc/00216/32711/

(C) 2014 The Japan Society of Fluid Mechanics and IOP Publishing Ltd

Printed in the UK

\title{
Vortex stability in a multi-layer quasi-geostrophic model: application to Mediterranean Water eddies
}

\author{
Carton Xavier ${ }^{1, *}$, Sokolovskiy Mikhail ${ }^{2}$, Ménesguen Claire ${ }^{4}$, Aguiar Ana ${ }^{3}$, Meunier Thomas ${ }^{1}$ \\ ${ }^{1}$ Laboratoire de Physique des Oceans, UBO/IFREMER/CNRS/IRD, Brest, France \\ 2 Institute of Water Problems of the RAS, Moscow, Russia \\ ${ }^{3}$ Instituto Dom Luiz, Universidade de Lisboa, Lisbon, Portugal \\ *Corresponding author : Xavier Carton, email address : xcarton@univ-brest.fr
}

\begin{abstract}
:
The stability of circular vortices to normal mode perturbations is studied in a multi-layer quasigeostrophic model. The stratification is fitted on the Gulf of Cadiz where many Mediterranean Water (MW) eddies are generated. Observations of MW eddies are used to determine the parameters of the reference experiment; sensitivity tests are conducted around this basic case. The objective of the study is two-fold: (a) determine the growth rates and nonlinear evolutions of unstable perturbations for different three-dimensional (3D) velocity structures of the vortices, (b) check if the different structure of our idealized vortices, mimicking MW cyclones and anticyclones, can induce different stability properties in a model that conserves parity symmetry, and apply these results to observed MW eddies. The linear stability analysis reveals that, among many 3D distributions of velocity, the observed eddies are close to maximal stability, with instability time scales longer than 100 days (these time scales would be less than 10 days for vertically more sheared eddies). The elliptical deformation is most unstable for realistic eddies (the antisymmetric one dominates for small eddies and the triangular one for large eddies); the antisymmetric mode is stronger for cyclones than for anticyclones. Nonlinear evolutions of eddies with radii of about $30 \mathrm{~km}$, and elliptically perturbed, lead to their re-organization into 3D tripoles; smaller eddies are stable and larger eddies break into 3D dipoles. Horizontally more sheared eddies are more unstable and sustain more asymmetric instabilities. In summary, few differences were found between cyclone and anticyclone stability, except for strong horizontal velocity shears.
\end{abstract}




\section{Introduction}

The Mediterranean Sea is an evaporation basin which produces salty waters; these waters are then exported into the Atlantic Ocean via the Straits of Gibraltar. There, they mix with North Atlantic Central Water while cascading down the continental slope (Madelain 1970). This Mediterranean Water (MW) outflow adjusts hydrostatically as two along-slope currents at 800 and $1200 \mathrm{~m}$ depths south of Portugal, near $36^{\circ} \mathrm{N}$ (Chérubin et al 2000). These currents flow across deep and narrow canyons which destabilize them (Chérubin et al 2007). This destabilization leads to the formation of anticyclonic eddies of MW (called meddies) and of shallower MW cyclones. Meddies are intensified between 800 and $1200 \mathrm{~m}$ depths, and have thermohaline radii (radius of maximal radial gradient in temperature and salinity) varying between 15 and $45 \mathrm{~km}$. MW cyclones are intensified (both in temperature, salinity and velocity) between 600 and $1000 \mathrm{~m}$ depths, but they also have a strong dynamical signature above this level; the few MW cyclones fully measured had a thermohaline radius of about $30 \mathrm{~km}$ (Carton et al 2002), Serra and Ambar 2002, Ambar et al 2008). MW cyclones and anticyclones often pair at the generation sites and these dipoles can remain coherent and drift cyclonically across the Gulf of Cadiz, before separating (Carton et al 2010). Observations at sea, as well as model results, indicate that both MW cyclones and anticyclones are relatively stable, but that most cyclones are found near the Iberian Peninsula (mostly in the Gulf of Cadiz) while meddies spread out across the Northeastern Atlantic Ocean (Menesguen et al 2012, Barbosa Aguiar et al 2013).

The present study is devoted to analyzing the conditions under which such vortices could become barotropically or baroclinically unstable. The settings for the study are idealized, though, with a five-layer quasi-geostrophic model having parameters (stratification, velocity and general structure of potential vorticity) fitted to the insitu data (Paillet et al 2002, Carton et al 2002, see subsection 2.2). The quasigeostrophic model allows detailed stability calculations but has parity symmetry. Thus, any difference in stability properties possibly found in this study would only result from the different three-dimensional structure of the eddies in velocity and in potential vorticity. Therefore, the terms cyclones and anticyclones hereafter refer more specifically to this 3D structure than to the sense of rotation of the vortices (again due to parity symmetry).

The paper is organized as follows: section 2 details the model and physical conditions of the study. Section 3 presents the linear stability results. In section 4 , the possible nonlinear evolutions of these eddies are shown and analysed. A discussion is conducted on the several possible evolutions of linearly unstable cyclones and anticyclones, depending on their 3D structure. Finally, a conclusion is provided and an appendix complements the linear stability study. 


\section{Model and physical settings}

\subsection{Quasi-geostrophic model}

Vortex stability is studied here in a five-layer quasi-geostrophic model. This choice is made to minimize the number of parameters for the model, while still allowing the representation of the vertical structure of MW eddies (see below for more details). This model has flat surface and bottom, and due to the small to medium size of these eddies, the f-plane approximation is used. No forcing nor dissipation is applied (except numerical biharmonic dissipation kept to a minimum in the nonlinear numerical model). The layerwise quasi-geostrophic equations are

$$
\frac{d q_{j}}{d t}=0, \quad j=1, \ldots, 5, \quad(\text { top to bottom })
$$

with layerwise potential vorticities (PV)

$$
q_{j}=\nabla^{2} \psi_{j}+F_{j, j-1}\left(\psi_{j-1}-\psi_{j}\right)+F_{j, j+1}\left(\psi_{j+1}-\psi_{j}\right),
$$

when applicable (the uppermost and lowermost PV layers have no coupling term with any layer above nor below them, respectively). The layer coupling coefficients are

$$
F_{j, j \pm 1}=(-1)^{ \pm 1} \frac{f_{0}^{2} \rho_{0}}{g H_{j}\left(\rho_{j \pm 1}-\rho_{j}\right)} .
$$

\subsection{Model parameters}

The model parameters are chosen as follows:

The Coriolis parameter is computed at $35^{\circ} \mathrm{N}$.

The choice of layer numbers and thicknesses is based on observations of the vertical structure of the potential vorticity anomaly of MW cyclones and anticyclones (anomalies with respect to the environment).

Meddies have maximum (negative) potential vorticity anomaly between 800 and $1300 \mathrm{~m}$ depths; two other (opposite signed) anomalies lie between 600 and $800 \mathrm{~m}$, and between 1300 and $1500 \mathrm{~m}$ depth, respectively (see Paillet et al 2002, Carton et al 2002). Enough observations at sea are available to assert that this vertically tripolar distribution of potential vorticity anomaly is characteristic of meddies.

Far fewer data are unfortunately available for cyclones, but one calculation of potential vorticity anomaly for a MW cyclone indicates a positive maximum between 600 and 1200 m depth, and secondary (weaker) extrema between 0 and 600, 1200 and 1800, 1800 and $2000 \mathrm{~m}$ depths, respectively (Carton et al 2002). Too few observations are available to firmly conclude on the predominance of vertical tripolar or dipolar distributions of potential vorticity anomalies for MW cyclones in general. The layer thicknesses thus chosen (to represent these potential vorticity structures) are summarized in table 1.

These observations at sea also provide density (see Carton et al 2002). An average density is obtained for all layers; with the layer thicknesses and the Coriolis parameter, these densities correspond to internal deformation radii of $32,14,8$ and $5 \mathrm{~km}$ 
respectively, which are in fair agreement with the measured values in the Gulf of Cadiz (away from the continental slope). The density differences between layers are summarized in table 1.

\begin{tabular}{|l||c|c|c|c|}
\hline Layer & $\begin{array}{c}\mathrm{H} \text { (cyclone) } \\
m\end{array}$ & $\begin{array}{c}\Delta \rho(1, \mathrm{l}+1) \text { (cyclone) } \\
\mathrm{kg} . \mathrm{m}^{-3}\end{array}$ & $\begin{array}{c}\mathrm{H} \text { (anticyc.) } \\
m\end{array}$ & $\begin{array}{c}\Delta \rho \text { (anticyc.) } \\
\mathrm{kg} \cdot \mathrm{m}^{-3}\end{array}$ \\
\hline 1 & 600 & 1.26 & 600 & 0.95 \\
\hline 2 & 600 & 0.125 & 200 & 0.35 \\
\hline 3 & 600 & 0.075 & 500 & 0.15 \\
\hline 4 & 200 & 0.062 & 200 & 0.1 \\
\hline 5 & 2000 & 0.0 & 2500 & 0.0 \\
\hline
\end{tabular}

Table 1. Thicknesses of layers for the cyclone and anticyclone models, and density differences between layers in both cases.

\subsection{Initial velocity profiles of the vortices}

For all cases studied in the main body of this study, the mean flow is circular and its azimuthal velocity is a power exponential of the radius

$$
V_{j}=U_{j} \frac{r}{2 R} \exp \left(-r^{\alpha} / R^{\alpha}\right),
$$

where $U_{j}$ is the layerwise velocity, $R$ is the vortex radius, and $\alpha=2,3$ controls the slope of the radial profile of velocity. This velocity profile corresponds to a radial profile of mean streamfunction given by

$$
\Psi_{j}=-2 U_{j} R \gamma\left(2 / \alpha,(r / R)^{\alpha}\right) / \alpha,
$$

where $\gamma(a, x)$ is the incomplete gamma function defined by

$$
\gamma(a, x)=\int_{0}^{x} t^{a-1} \exp (-t) d t
$$

In the simple case where $\alpha=2$, one recovers a Gaussian profile for $\Psi_{j}$.

Again, this choice is motivated by observations of MW eddies. In particular, Paillet et al (2002) fitted a Rayleigh profile (i.e. that given by equation (4) with $\alpha=2$ ) on a measured radial profile of meddy velocity; this fit was fairly accurate close to the meddy center (where the velocity increases linearly with the radius); in the outer part of the meddy, it was more difficult to fit a single curve, and both the Rayleigh profile, or smoother profiles (like the Rankine one in $1 / r$ ) or steeper profiles (like the cubic exponential profile, corresponding to equation (4) with $\alpha=3$ ) were acceptable.

Meddies (MW anticyclones) have maximal velocities on the order of -0.2 to -0.5 $\mathrm{m} / \mathrm{s}$, at 20-30 km distances from their axis and at 800 to $1200 \mathrm{~m}$ depths (from experiments at sea; see for instance Paillet et al 2002). Though far fewer observations of cyclones exist, MW cyclones have maximal velocities on the order of 0.15 to $0.5 \mathrm{~m} / \mathrm{s}$ at about 20-30 km from their axis and at 500 to $1100 \mathrm{~m}$ depths; note that, in MW 

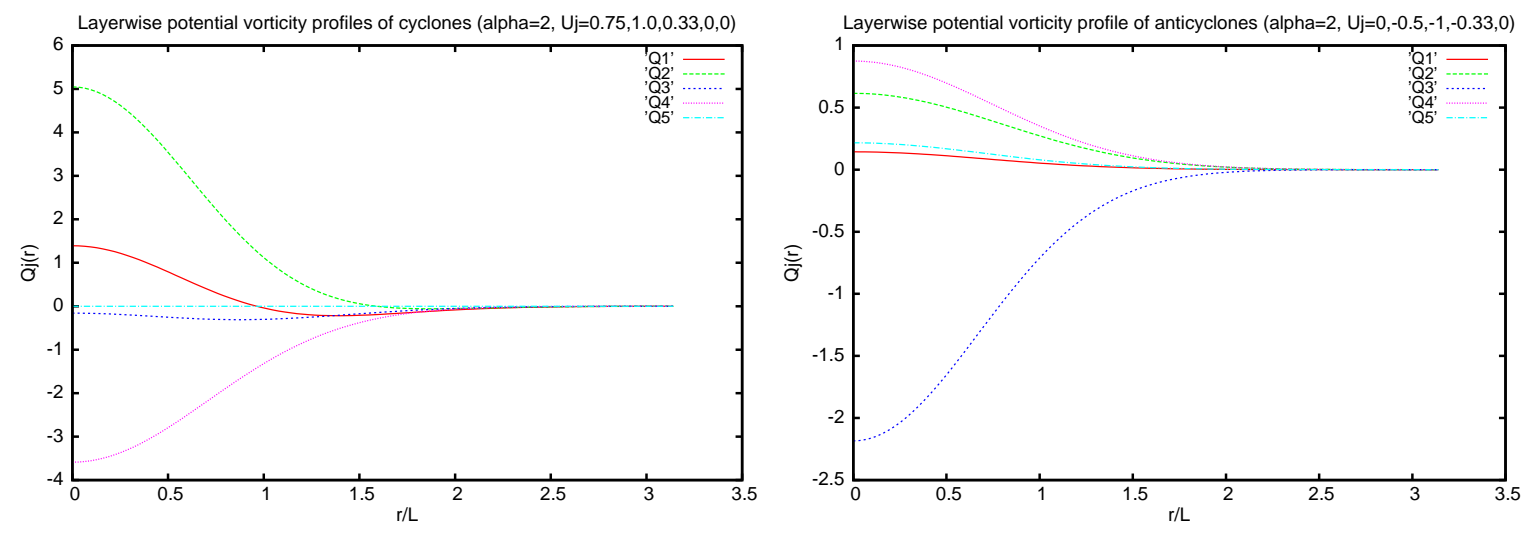

Figure 1. Radial profiles of potential vorticity in the model for cyclones (left), and for anticyclones (right), in the reference case, with $\alpha=2$.

cyclones, the average velocity in the upper $600 \mathrm{~m}$ of the ocean, is only slightly weaker than that in the $600 \mathrm{~m}$ just below.

Hereafter, we choose the length scale as $R=30 \mathrm{~km}$ (i.e. the scaled radius is unity for our vortices) and the velocity scale is $U_{\max }=0.3 \mathrm{~m} / \mathrm{s}$. From the length and velocity scales, we obtain a time scale of about 1.2 days.

From the analysis of data at our disposal, and setting a maximum velocity in layer 2 for the cyclones, and in layer 3 for the anticyclones, the ratio of layerwise velocities leads to the following values for the $U_{j}$ :

$U_{1}=0.75, U_{2}=1, U_{3}=0.33, U_{4}=0, U_{5}=0$ for the cyclone, and $U_{1}=0, U_{2}=$ $-0.5, U_{3}=-1, U_{4}=-0.33, U_{5}=0$ for the anticyclone.

Therefore, we define hereafter as "reference cases" the cyclones and anticyclones, having these values of maximal layerwise velocity, and a Gaussian radial profile of mean streamfunction (corresponding to equations (4)-(6) with $\alpha=2$ ).

On figure 1, we plot the radial profiles of potential vorticity in the model for the reference cases of cyclone and anticyclone. We note that the sign reversal of the radial gradient of potential vorticity occurs mainly along the vertical axis (from layer to layer), and more weakly along the radial axis (for cyclones). Thus we anticipate that baroclinic instability will be predominant for these vortices with $\alpha=2$, though barotropic instability can also affect them (as was shown by Carton and McWilliams (1989), for two-dimensional Gaussian vortices). Barotropic instability will be more efficient on cubic exponential vortices $(\alpha=3)$ which have a steeper radial profile of velocity.

To account for the various distributions of velocity in meddies or in MW cyclones in the ocean, the ratios of layerwise velocities will be varied around the reference case in this study. This will allow an investigation of the effects of such variations on the stability of these vortices. Depending on their velocity distributions and with the given stratification, the structure in potential vorticity of the cyclones and anticyclones in our model, can be vertically dipolar or tripolar, initially. At finite times, instability can lead to the breaking of potential vorticity poles, leading to more complex three-dimensional 
structures in potential vorticity.

\section{Linear stability}

\subsection{Theory and numerical method}

For the linear stability study, the layerwise quasi-geostrophic equations (1)-(3) are linearized around the axisymmetric mean flow (equations (4) and (5)), and normal modes of the form

$$
\psi_{j}^{\prime}(r, \theta, t)=\phi_{j}(r) \exp [i l(\theta-c t)]
$$

are used as perturbations (where $j=1, \ldots, 5$ is again the layer index). The phase speed of these modes is $\operatorname{Re}(c)=c_{r}$ and their growth rate is $l \operatorname{Im}(c)=l c_{i}$. When the radius is discretized over a finite number of steps $\left(N_{r}\right)$ in each layer, the linear problem is of the form

$$
A \phi_{j}\left(r_{k}\right)=c B \phi_{j}\left(r_{k}\right), \quad k=1, \ldots, N_{r}, \quad j=1, \ldots, 5,
$$

where $A$ and $B$ are $5 N_{r} \times 5 N_{r}$ matrices depending on the mean flow velocity and potential vorticity gradient. The boundary condition at infinity (in radius) for $\phi_{j}$ is convergence towards zero. Due to computing time constraints, $N_{r}=80$ is chosen and the corresponding spatial step for the linear stability study is $\Delta r=0.04$. The relative differences in growth rates between $N_{r}=60$ and $N_{r}=80$, tested with the anticyclones of figure 3 , is on the order of $0.1 \%$. For a few cases (calculation of the spatial structure of the eigenmodes, or comparison of growth rates between the linear and nonlinear models), an even higher resolution $\left(N_{r}=120\right)$ is used. Equation (8) is solved in finite differences via a generalized eigenvalue/eigenvector solver (LAPACK library).

In the appendix, we analyse the linear stability of different velocity and potential vorticity profiles (other than power exponential), to highlight how vortex stability depends on the details of the radial profile of velocity.

\subsection{Influence of the vortex radius on its linear instability}

Firstly, we consider vortices with Gaussian radial profiles of mean azimuthal velocity (equations (4) and (5) with $\alpha=2$ ). The vertical distribution of velocity is that of the reference case.

Figure 2 shows the variations of the growth rates with the vortex size for the cyclones and anticyclones, for the gravest modes $(l=1,2,3)$.

A common feature of all these figures is that the growth rates of the triangular mode $l=3$ become large only for wide vortices (with radii on the order of $75 \mathrm{~km}$ ). The elliptical mode $l=2$ dominates for Gaussian vortices $(\alpha=2)$ with radii between about 30 and $75 \mathrm{~km}$. This predominance is more clear for anticyclones, for which it starts at radii of about $15 \mathrm{~km}$. The predominance of the elliptical mode is much more striking for cubic exponential vortices, which are more sensitive to barotropic instability. This predominance of the elliptical mode for barotropically unstable vortices has already been 

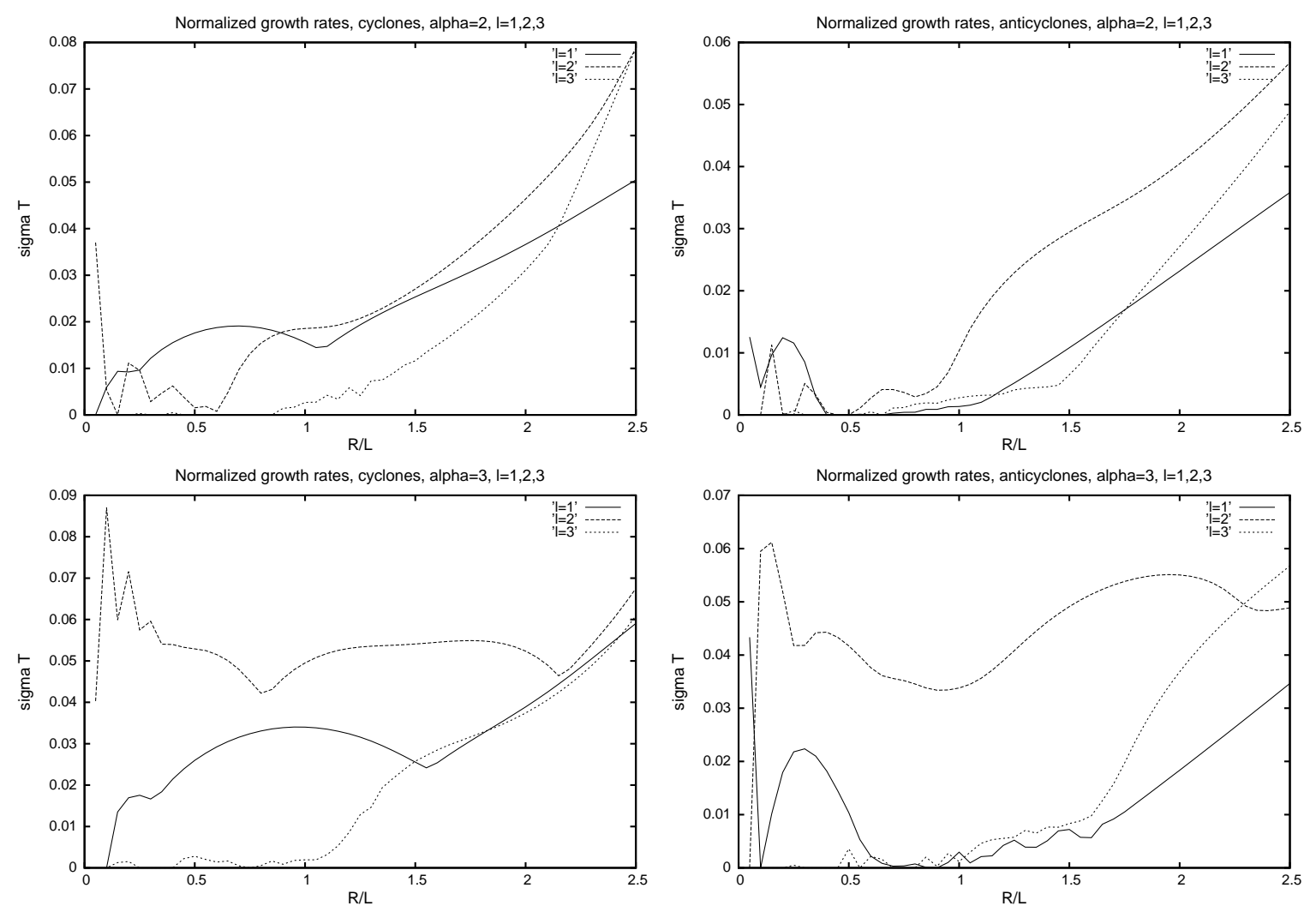

Figure 2. Normalized growth rates of normal modes, with respect to the normalized vortex radius $R / L$ for Gaussian cyclones (top left), Gaussian anticyclones (top right), cubic exponential cyclones (bottom left) and cubic exponential anticyclones (bottom right) and for wavenumbers $l=1,2,3$.

noticed in the 2D case (Carton and McWilliams (1989)).

The antisymmetric mode is clearly more unstable than the elliptical mode only for Gaussian cyclones with radii between 10 and $25 \mathrm{~km}$ (and for anticyclones with radii between 5 and $10 \mathrm{~km})$. For vortex radii below $5 \mathrm{~km}(R / L<0.17)$, the quasi-geostrophic model results may differ from the real ocean dynamics which involve strong vertical motions at these submesocales. A primitive equation model would be necessary to study the stability of small vortices.

As a first conclusion, the elliptical mode dominates in the instability of small to medium size Gaussian anticyclones, while both antisymmetric and elliptical modes grow on small to medium size Gaussian cyclones.

On the contrary, for both cyclones and anticyclones with a cubic exponential profile of velocity, the elliptical mode of instability dominates that of the antisymmetric mode for nearly all vortex sizes. 

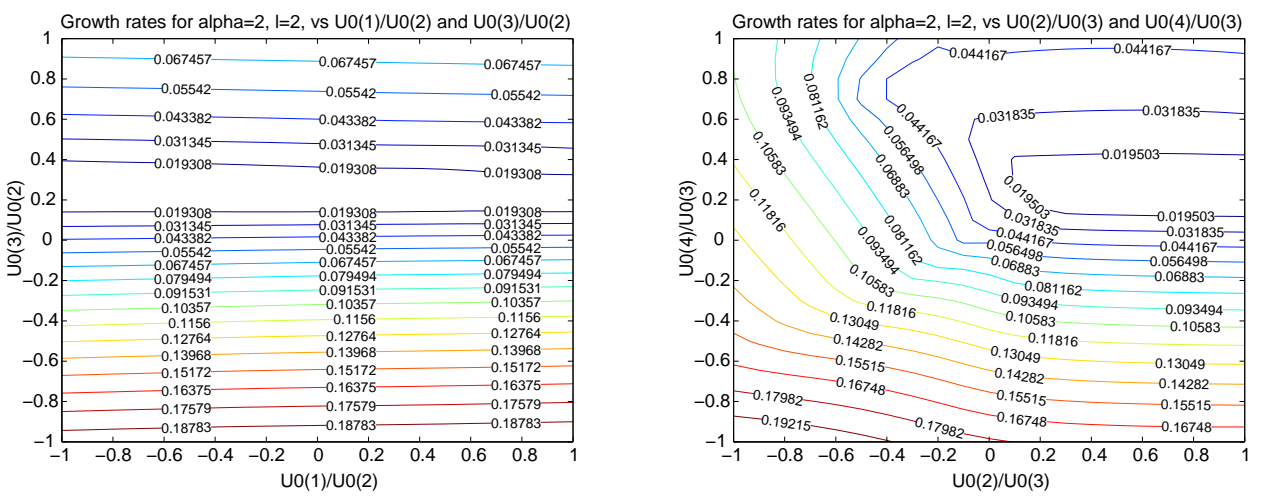

Figure 3. Isolines of normalized growth rates of elliptical normal modes, in the $U_{1} / U_{2}, U_{3} / U_{2}$ plane for Gaussian cyclones (left), and in the $U_{2} / U_{3}, U_{4} / U_{3}$ plane for Gaussian anticyclones (right).

\subsection{Influence of the layerwise velocity ratios on linear stability}

Figure 3 presents the growth rates of elliptical normal modes (with $l=2$ ) for Gaussian cyclones and anticyclones $(\alpha=2)$ in the $\left(U_{1} / U_{2}, U_{3} / U_{2}\right)$ and $\left(U_{2} / U_{3}, U_{4} / U_{3}\right)$ planes respectively; thus, here we vary the ratios of maximal layerwise velocities of the mean flow. For cyclones and anticyclones with $\alpha=2$, minimal growth rates are about 0.01 and growth time scales about 120 days, for an elliptical perturbation. This occurs in particular for the reference case.

For cyclones, growth rates increase if $U_{3} / U_{2}$ increases or decreases from the reference value (0.33). This increase is related to the vertical structure of cyclones in potential vorticity which depends on this velocity ratio. On the contrary, the growth rates are less dependent on the ratio $U_{1} / U_{2}$. Note that the maximal growth rates, on the order of 0.2 - or growth time scales of 6 days - are obtained for strongly baroclinic cyclones. This is consistent with the fact that for cyclones with $30 \mathrm{~km}$ radii, the instability is mostly baroclinic (see also figure 2 showing that growth rates increase with $R / L$, for cyclones with radii around $30 \mathrm{~km}$ ).

For anticyclones, growth rates are sensitive to $U_{4} / U_{3}$ and to $U_{2} / U_{3}$ because changes in both ratios modify the vertical structure in potential vorticity. Note that the largest growth rates, again corresponding to growth time scales of about 6 days, are obtained for strongly baroclinic vortices.

For vortices with cubic exponential velocity profiles, the maximal growth rates of elliptical perturbations are comparable to those for Gaussian vortices, while the minimal growth rates are twice as large as those for Gaussian vortices (see figure 4). This is also noticed on figure 2 for the reference case.

Finally, we present the geometrical structure of the normal mode perturbation for the Gaussian anticyclone in the reference case (see figure 5). The phase of the perturbation increases from layer 4 to layer 3 and decreases back to layer 2, contrary to the mean velocity. This variation is characteristic of baroclinic instability. The modes in layers 2 , 3,4 are strongly influenced by the intense mean flow via a noticeable change in phase 

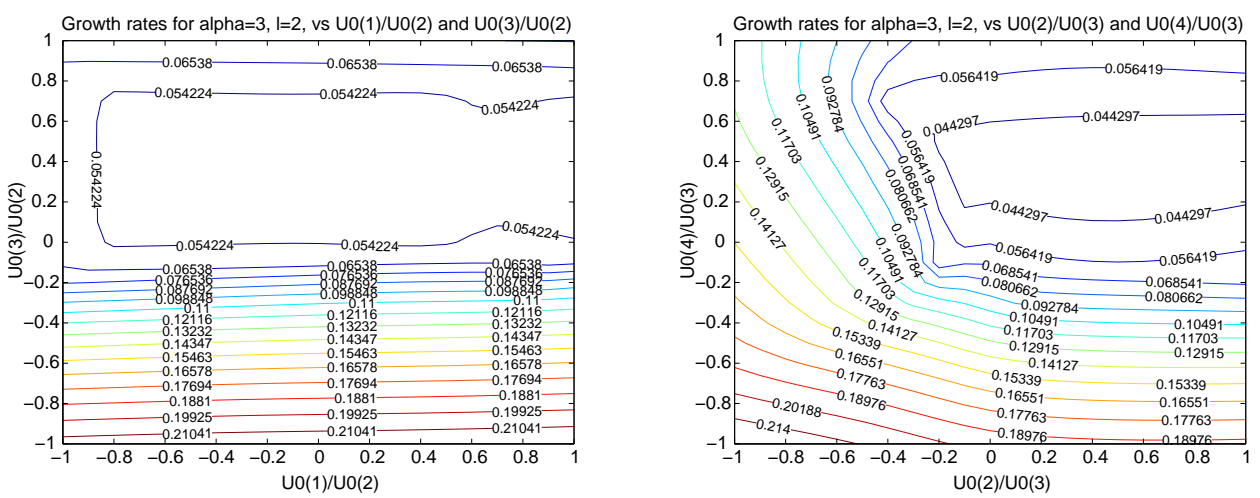

Figure 4. Isolines of normalized growth rates of elliptical normal modes, in the $U_{1} / U_{2}, U_{3} / U_{2}$ plane for cubic exponential cyclones (left), and in the $U_{2} / U_{3}, U_{4} / U_{3}$ plane for cubic exponential anticyclones (right).
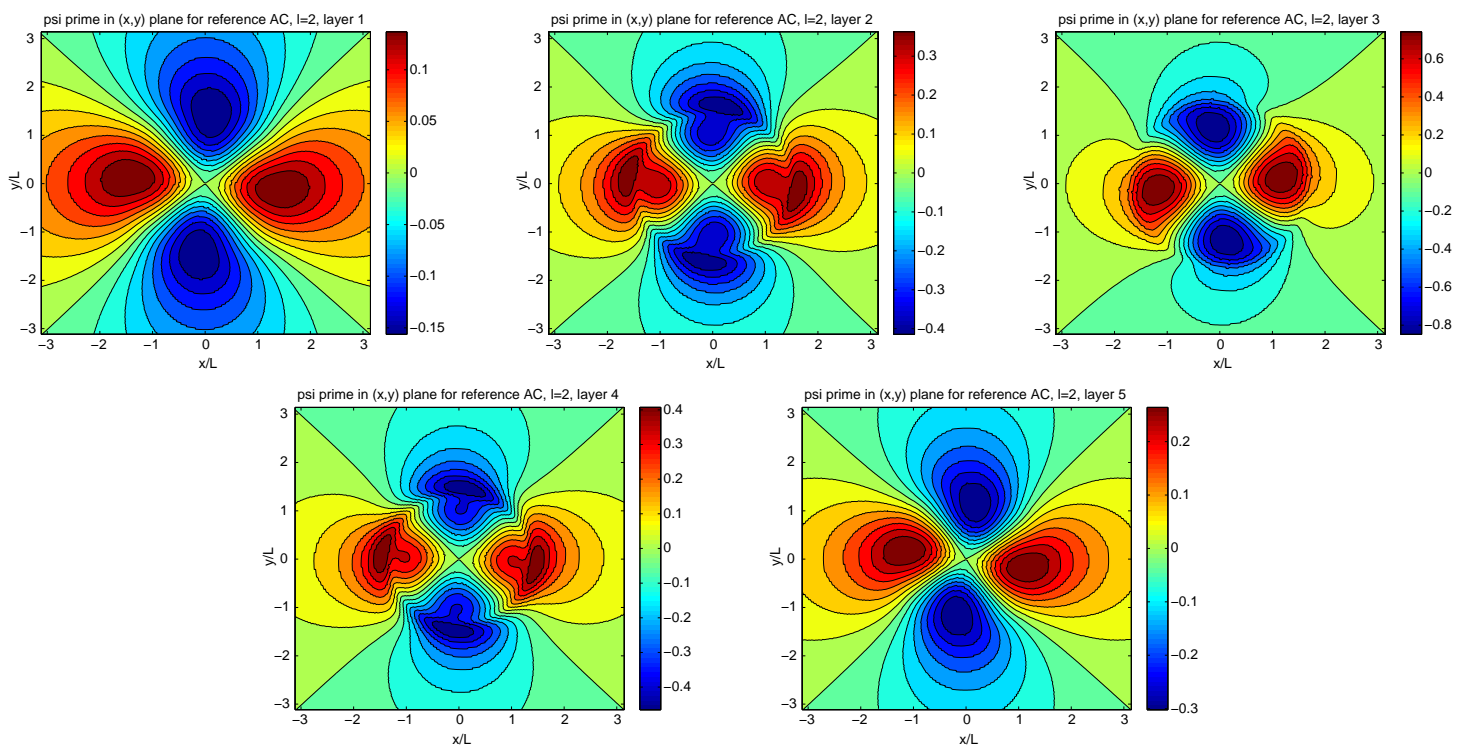

Figure 5. Streamlines of the most unstable normal mode perturbation for the Gaussian anticyclone $(\alpha=2)$ in the reference case; layers 1 to 5 (left to right, then top to bottom) are displayed.

at finite radius. This feature is characteristic of a critical layer in the mean flow (where the angular rate for the mean flow $V_{j} / r$ equals the phase speed $c_{r}$ of the $l=2$ wave). By approximating the radii of phase change to $r=1.5$ in layer 2 and to $r=1.3$ in layer 4 , we obtain $V_{j} \approx 0.04$ in both layers, and therefore $V_{j} / r \approx 0.027,0.031$ at these radii, while $c_{r} \approx 0.027$. 


\section{Nonlinear evolutions}

\subsection{Numerical method}

To study the nonlinear evolution of the unstable vortices, a small-amplitude, modal perturbation is initially added to their circular streamfunction (equations (4)-(6) and simulations are run in a numerical model of the complete quasi-geostrophic equations (1)-(3). This numerical model is based on a truncated, pseudo-spectral projection of the equations (in space), with a Heun (mixed Euler-leapfrog) scheme in time. The time step is constrained by the Courant-Friedrichs-Lewy condition. The number of grid points is $256 \times 256$ in the horizontal plane (the simulations performed at 512x512 resolution show very similar evolutions).

To avoid enstrophy accumulation at small scale, a weak biharmonic viscosity is added to the model. It has been checked that this weak dissipation does not alter the outcome of the simulations (which are performed on much shorter time scales than the viscous timescale).

As a consistency check between these nonlinear simulations, and the linear stability analysis, simulations are performed with a small time interval to calculate the growth rates of the elliptical mode in several cases. For the anticyclone in the reference case, the linear growth rate is $\sigma=l c_{i}=0.011$ while that obtained in the nonlinear simulation is $\sigma=0.013$. For the unstable anticyclone shown on figure 7, the linear and nonlinear growth rates are $\sigma=0.054$. For the unstable anticyclone shown on figure 8 , the linear and nonlinear growth rates are $\sigma=0.024$. Finally, for the unstable cyclone of figure 11 , these values are respectively $\sigma=0.088$ and $\sigma=0.084$. The relative error on the growth rates is on the order of $1-5 \%$ (at most $15 \%$ for very weak growth rates). The correspondence is thus fair.

Firstly, the vortex radius is varied and the evolutions are characterized. In this study, only vortices initially perturbed elliptically are studied. The stability for other initial perturbations should be addressed in another article. In the second part of this section, the layerwise velocity ratios are modified to determine the possible nonlinear regimes for various types of vortices.

\subsection{Influence of vortex size}

For vortices in the reference case, and for an elliptical mode of deformation $(l=2)$, nonlinear stability is observed when the vortex radius is smaller than 0.75 times the first internal radius of deformation, $R<0.75 R_{d}$, for cyclones and anticyclones (see figure 6 ). Unstable, perturbed vortices break into two three-dimensional dipoles if $R>1.25 R_{d} \S$.

$\S$ We call three-dimensional dipoles - or tripoles - vortex structures which have opposite-sign vorticity poles in several layers; 3D dipoles, resp. tripoles, will have positive potential vorticity poles in some layer coupled with negative vorticity poles in other layers, leading to global translation, resp. rotation 
ANTICYCLONES (ALPHA $=2)$

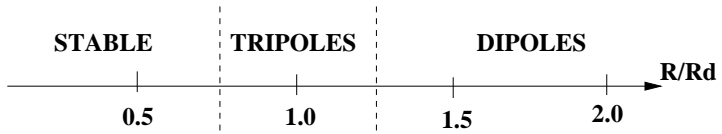

CYCLONES $\quad($ ALPHA $=2)$

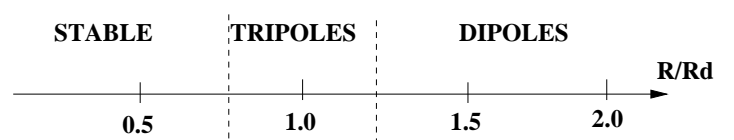

ANTICYCLONES (ALPHA $=3$ )

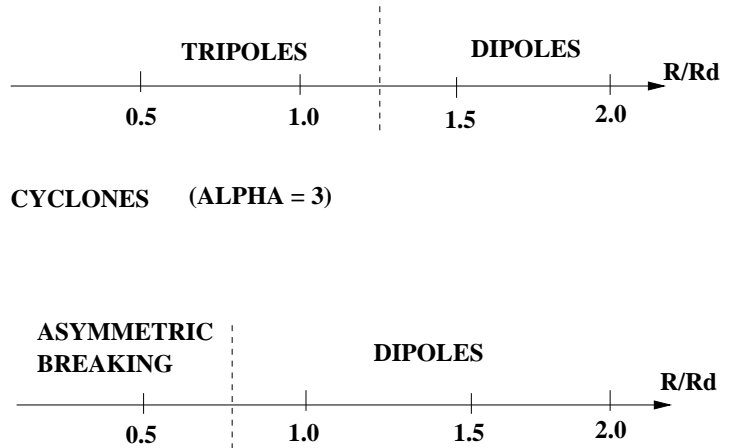

Figure 6. Nonlinear regime diagram for cyclones and anticyclones with Gaussian (left) or cubic exponential profiles (right), with respect to the vortex size, using the layerwise distribution of velocity of the reference case
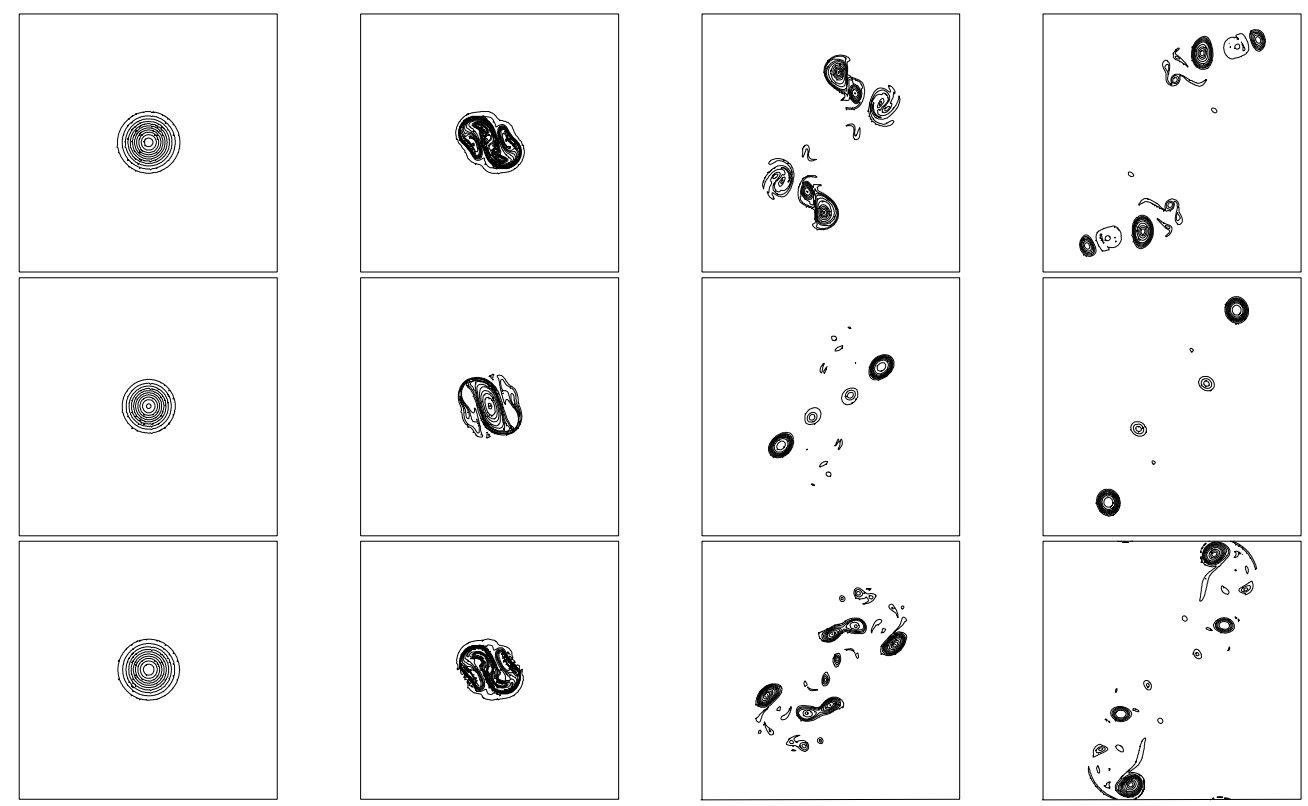

Figure 7. Time evolution (from left to right) of potential vorticity contours for an unstable Gaussian anticyclone with $l=2, R=1, U_{2} / U_{3}=0$ and $U_{4} / U_{3}=0$. The vortex undergoes baroclinic dipolar breaking. Layers $2,3,4$ are shown from top to bottom. Contour intervals are $\Delta q_{2}=0.2, \Delta q_{3}=0.4$ and $\Delta q_{4}=0.5$.

Between these two bounds, perturbed vortices stabilize nonlinearly as three-dimensional tripoles, again for both polarities. This can be understood in terms of baroclinic instability for which shorter waves grow when the characteristic scale of the mean flow increases.

For vortices with cubic exponential velocity profiles, the nonlinear regimes of anticyclones are similar to those for Gaussian vortices, whereas the regimes for cyclones indicate a stronger instability: for $R<0.75 R_{d}$, asymmetric breaking of the vortex is 

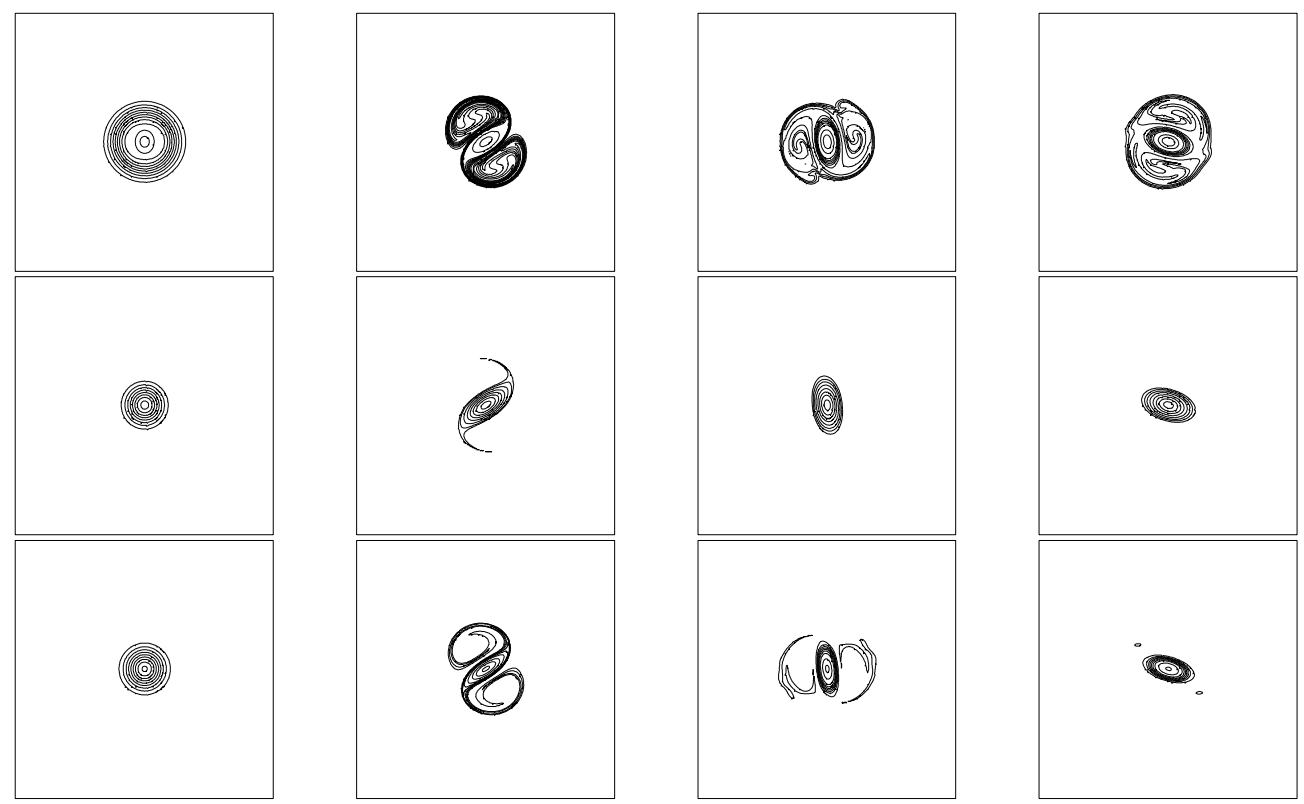

Figure 8. Time evolution (from left to right) of potential vorticity contours for an unstable Gaussian anticyclone with $l=2, R=1, U_{2} / U_{3}=0.5$ and $U_{4} / U_{3}=0.5$. Layers 2, 3, 4 are shown from top to bottom. The vortex undergoes stabilization into a Y-shaped baroclinic (3D) tripole. Contour intervals are $\Delta q_{2}=0.03, \Delta q_{3}=0.3$ and $\Delta q_{4}=0.2$.

observed after more than 10 turnover periods of the baroclinic (3D) tripole; larger cyclones break into baroclinic (3D) dipoles (again for elliptical perturbations; see figure 6).

Two main regimes, namely baroclinic dipolar breaking and the formation of a baroclinic tripole, are illustrated on figures 7 and 8 . The difference between these two regimes lies in particular in the distribution of potential vorticity out of the central elliptical core:

- when baroclinic dipolar breaking occurs (figure 7), the peripheral lobes of vorticity are strong in each layer. They induce a large deformation on the core vortex. Thus, the rotation of this central ellipse slows down, and each peripheral lobe moves towards each end of the elliptical core. This leads, in the case of dipolar breaking, to the coupling of each peripheral lobe with half of the core vortex, thereby amplifying again its aspect ratio. Therefore a baroclinic dipole is formed.

- in the case of baroclinic tripole formation (figure 8), these 3D tripoles can assume different shapes depending on the initial distribution of potential vorticity in the circular vortex. When the maximum vertical gradient of potential vorticity lies between layers 2 and 3, the tripoles have satellites only above the core (Y-shaped tripoles; case of figure 8); conversely, satellites will lie below the core for strong vertical gradients of potential vorticity at these depths ( $\Lambda$-shaped tripoles); finally, some tripoles will have satellites in both layers (X-shaped tripoles), if the gradients above and below the core are comparable. 


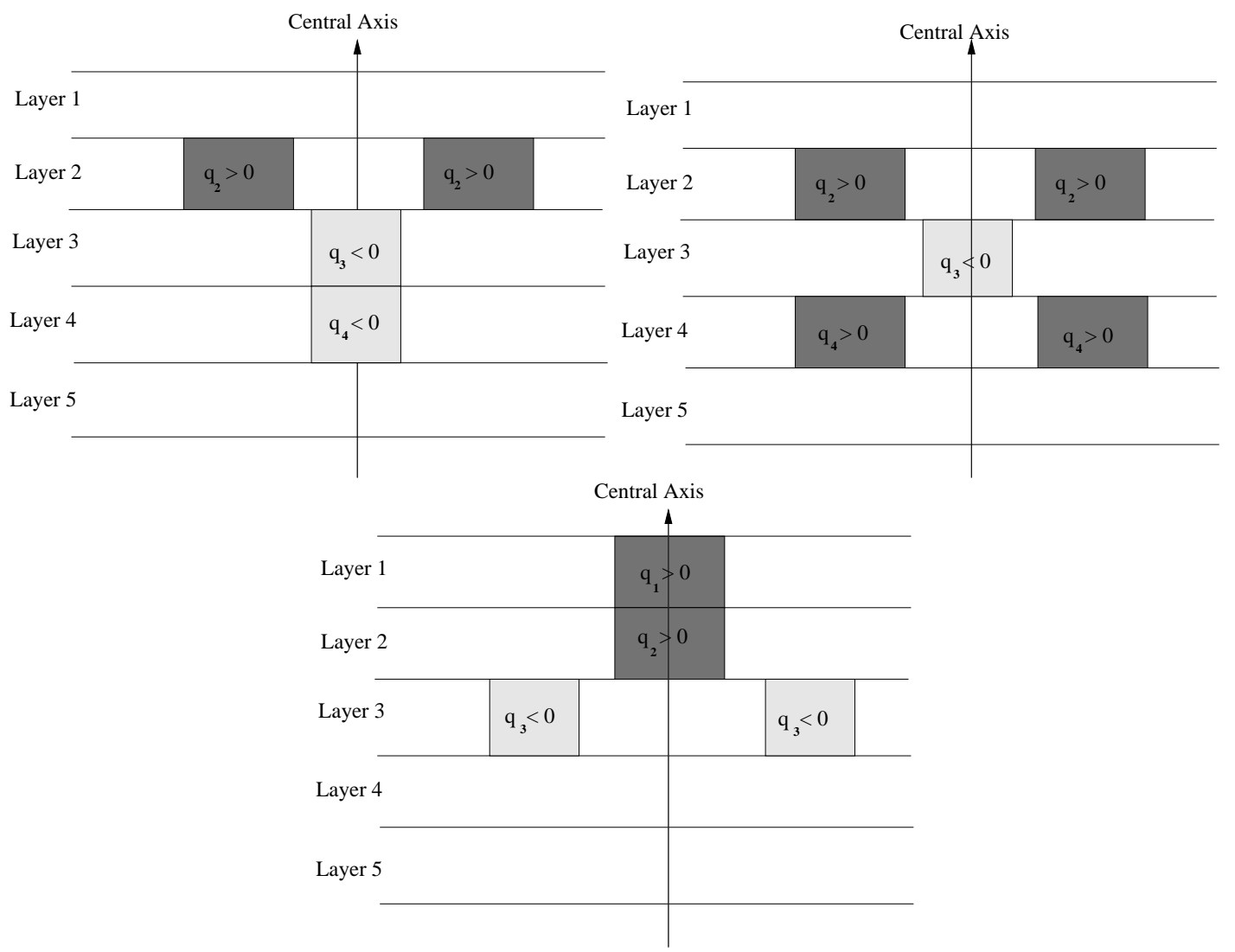

Figure 9. Schematic representation of the $\mathrm{Y}$ tripole (top left), of the $\mathrm{X}$ tripole (top right) and of the $\Lambda$ tripole (bottom).
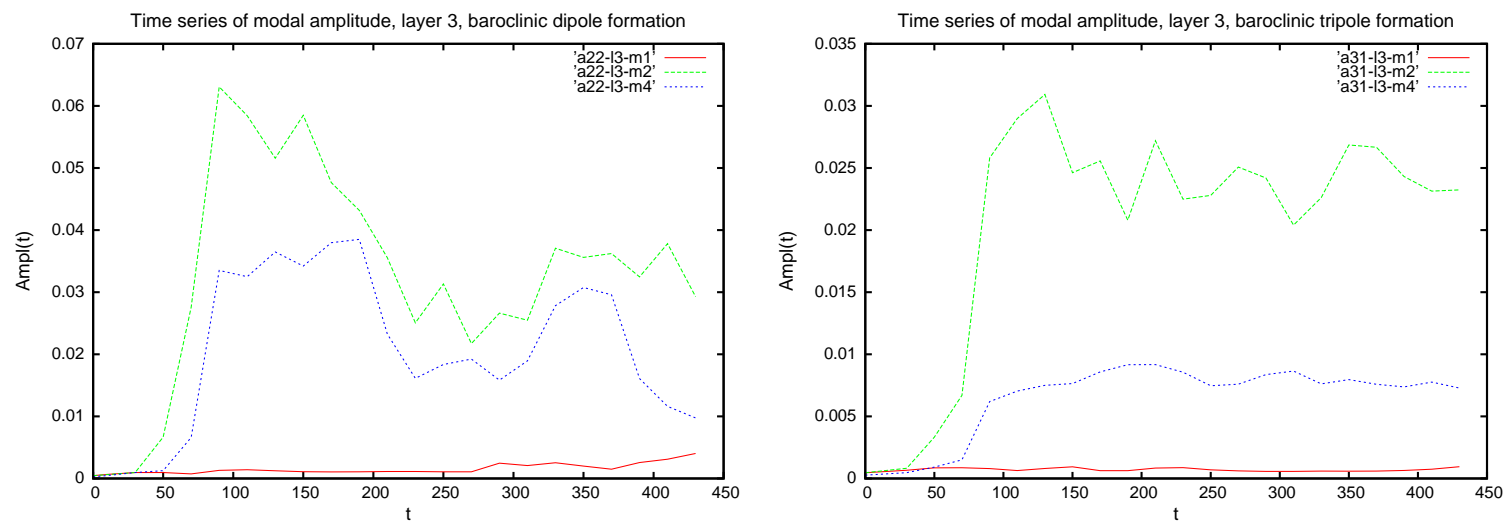

Figure 10. Time evolution of the Fourier amplitudes of modes $l=1,2,4$ in the middle layer, for the dipolar breaking of a Gaussian anticyclone with an elliptical perturbation (left) and for a nonlinear stabilization into a baroclinic tripole (right). $A m p l(t)$ indicates the modal amplitude on the ordinate axis.

Figure 9 presents a schematic representation of these various forms of tripoles.

In figure 10, we show the Fourier analysis (in angle) of the perturbation for dipolar breaking and for tripole formation; the Fourier coefficients in the middle layer of each vortex are plotted versus time. In both cases, the antisymmetric mode $l=1$ remains 

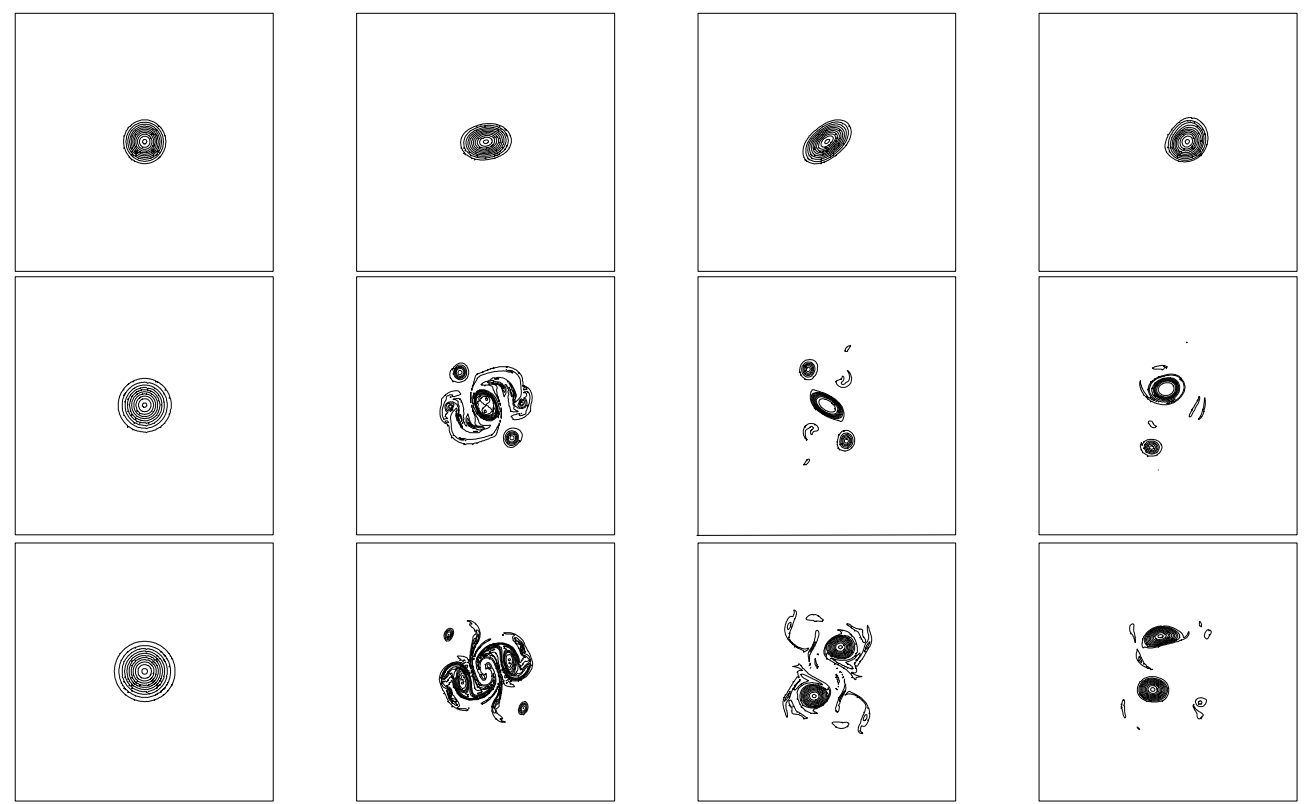

Figure 11. Time evolution (from left to right) of potential vorticity contours for an unstable Gaussian cyclone with $l=2, R=1, U_{1} / U_{2}=-0.75$ and $U_{3} / U_{2}=-0.25$. The vortex undergoes asymmetric destabilization. Layers $1,2,3$ are shown from top to bottom. Contour intervals are $\Delta q_{1}=0.1, \Delta q_{2}=0.4$ and $\Delta q_{3}=0.5$.

weak (growing from numerical noise); mode $l=2$ is dominant but grows to a weaker amplitude when nonlinear stabilization occurs than when dipolar breaking prevails. Also, the first harmonic mode $l=4$ is much weaker in the case of nonlinear stabilization. Indeed, intense short waves are necessary for the splitting of vortices.

Finally, another regime is shown which occurs mostly for vortices with cubic exponential profiles of velocity, but also for Gaussian cyclones, with strong vertical shears of velocity (figure 11). In this evolution, the filaments which are initially ejected from the elliptical core vortex undergo slightly unequal deformation (due to the presence of mode $l=1$ ), and dissipation. This in turn leads to slightly asymmetric strengths of the peripheral lobes around which these filaments can wrap. These unequal strengths of the satellite vortices lead to a vacillation of the baroclinic tripolar (3D) structure, and to mode $l=1,2,3$ deformations of the contour of the core vortex. The tripole extends and bends, and often, satellites can merge. In its final state, the multi-layer vortex compound can still be rotating (as long as mode $l=2$ still dominates) or it can be propagating (if mode $l=1$ prevails). This asymmetric evolution is made possible by the non negligible growth rate of mode $l=1$ for vortices with radius close to the first deformation radius. 

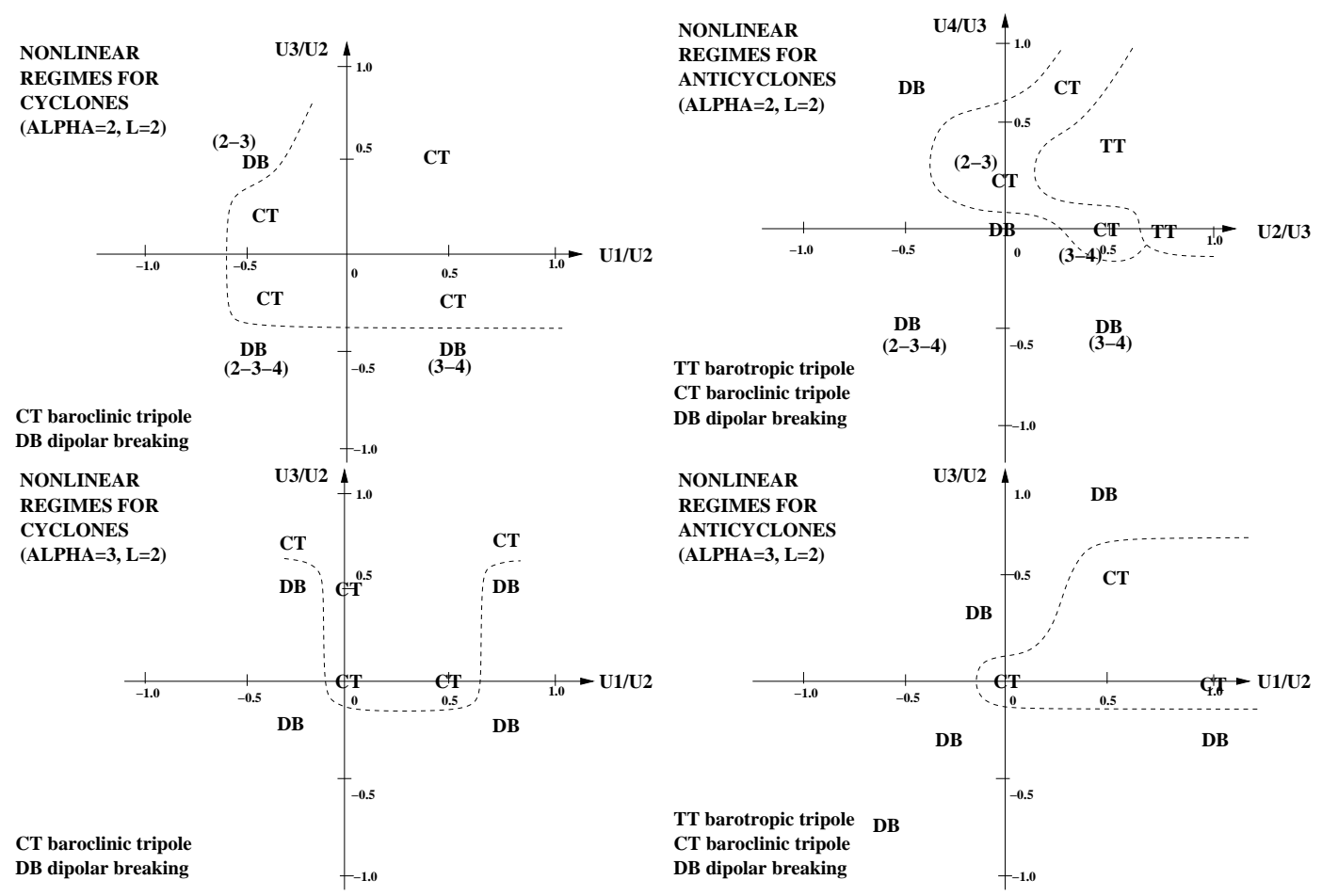

Figure 12. Regime diagram of nonlinear evolutions in the parameter plane of the layerwise velocity ratios for cyclones and anticyclones with Gaussian or cubic exponential profiles of velocity.

\subsection{Influence of the ratios of layerwise velocities}

Now we present maps of nonlinear regimes in the parameter space of layerwise velocity ratios (see figure 12). To obtain these maps, about 125 numerical simulations were performed.

Clearly, Gaussian cyclones and anticyclones tend to form baroclinic tripoles (of any of the X, Y or $\Lambda$ types) when the initial vortex is co-rotating, or slightly counter-rotating, in its main three layers. Due to their dipolar or tripolar vertical structure in potential vorticity, cyclones or anticyclones form 3D baroclinic tripoles which have opposite signs of vorticity in their main three layers, that is, with a core in the central layer (layer 2 for cyclones and layer 3 for anticyclones) and with satellites of opposite signed vorticity, in the layers above and/or below the core. The predominance of dipolar breaking when vortices are strongly counter-rotating, or the formation of baroclinic tripoles in other cases, is indicative of a dominant baroclinic instability.

Only when they are strongly co-rotating can Gaussian anticyclones form barotropic tripoles in potential vorticity; these tripolar structures have a core and satellites in all layers (2 to 4 ) and the cores are like signed in these layers. This is then the result of a dominant barotropic instability.

On the contrary, for cubic exponential profiles of velocity, the horizontal velocity 
shear is much stronger; thus barotropic instability is more influential on the nonlinear evolution of vortices. This is manifested by the more frequent occurrence of horizontal breaking of vortices (see the regime diagram in the velocity ratio plane in figure 12). This occurs obviously for more barotropic vortices. The occurence of asymmetric breaking is more frequent than for Gaussian vortices too, affecting both cubic exponential cyclones and anticyclones in the long run, whereas mostly Gaussian cyclones break asymmetrically. Note that cubic exponential cyclones with $U_{1} / U_{2}=0.75$ and $U_{3} / U_{2}=0.33$ can break whereas cubic exponential anticyclones with $U_{2} / U_{3}=0.5$ and $U_{4} / U_{3}=0.33$ stabilize.

\section{Conclusions}

The linear stability analysis of circular vortices in a five-layer quasi-geostrophic model indicates growth time scales of 10 to 100 days for normal mode perturbations (case of an elliptical deformation). This elliptical mode dominates for vortices with size similar to the first internal radius of deformation (about $30 \mathrm{~km}$ ), more strongly for anticyclones than for cyclones. The antisymmetric mode is more unstable for small vortices in particular, and the triangular one arises on large vortices. Note though that the antisymmetric mode can be unstable for a wide range of vortex sizes.

For vortices with a Gaussian profile of velocity, baroclinic instability is dominant when the vortex radius is similar to, or larger than, the first internal deformation radius. Barotropic instability prevails for smaller eddies. Vortices with cubic exponential velocity profiles are more sensitive to barotropic instability.

Eddies with velocity and size characteristics similar to those of meddies or of MW cyclones are weakly unstable (growth time scales of 2 to 3 months, see also NGuyen et al (2012) who find dominance of mode $l=1$ for $R<R_{d}$ and of mode $l=2$ for $R>R_{d}$ in general agreement with our study).

Note also (see appendix below), that for different radial profiles of mean velocity, the variation of the normal mode growth rates with the ratios of layerwise mean velocities are similar, for all cyclones on the one hand, and for all anticyclones on the other. We also show in this appendix that vortices with Gaussian streamfunction have similar instability properties to vortices with Gaussian relative vorticity, and that they are more unstable than vortices whose mean azimuthal velocity decays exponentially at large radii.

Nonlinear numerical simulations show that baroclinic instability dominates the evolution of these unstable vortices when they have a Gaussian profile of velocity, whereas barotropic instability can be prevalent for cubic exponential velocity profiles or when the eddies are strongly co-rotating.

When barotropic instability dominates, the formation of barotropic tripoles is possible for co-rotating Gaussian anticyclones, whereas horizontal dipolar breaking is observed for cubic exponential co-rotating vortices. 
When baroclinic instability dominates, the nonlinear evolution of the vortices (of both polarities) is either the formation of baroclinic (3D) tripoles, when the instability is not too intense, or the breaking of the vortex into baroclinic (3D) dipoles, for stronger instabilities.

Baroclinic tripoles can form under various configurations, depending on the details of the $3 \mathrm{D}$ distribution of potential vorticity and on the vertical velocity shear. The elliptical core of these tripoles lies in the central layer of the eddy while opposite signed satellites form either above the core (Y-shaped tripoles), beneath it ( $\Lambda$-shaped tripoles), or in both layers (X-shaped tripoles).

A Fourier analysis of the time-varying perturbation has shown that tripole formation is accompanied by the stabilization of the elliptical mode while its first harmonic grows only moderately. On the contrary, dipolar breaking is due to cooperative nonlinear interactions which amplify both the fundamental elliptical mode and its first harmonic, up to large amplitudes.

It has also been found that asymmetric breaking can prevail on the long run, often after a stage of 3D tripole formation, and essentially for cyclones, or for counter-rotating, cubic exponential vortices. This evolution is due to the progressive amplification of the antisymmetric mode $(l=1)$, both via linear and nonlinear effects, leading to a lateral vacillation and to a bending of the tripole which may result in the merger of the satellites.

To compare the results of our simple model with observations, we can note that cyclones and anticyclones in the reference case are most unstable to the elliptical $(l=2)$ and antisymmetric $(l=1)$ perturbations, which have very long growth time scales (on the order of 3 months). In our nonlinear model, we showed that the formation of tripoles (and thus the long-term preservation of the central vortex cores) is an often-found evolution in the velocity-ratio parameter plane. This compares favorably with observations :

Tychensky and Carton (1998) showed that the upper potential vorticity pole of meddies Hyperion, Ceres and Encelade were split into two parts. It is not unreasonable to attribute this splitting to the interaction of these meddies with the nearby Azores Current, which exerts a shear on these eddies (and thus creates mode 1 and 2 deformation).

Carton et al (2010) also found split upper potential vorticity poles in the interaction of two meddies, southwest of Portugal. Again the shear exerted by each vortex on the other may be held responsible for this splitting.

Menesguen et al (2012) showed the tripolar structure of a meddy several months after its generation.

Finally, our model has shown only a fairly modest difference in stability between cyclonic and anticyclonic vortex initializations. The difference in stability between cy- 
clones and anticyclones is mostly related to the possibility of asymmetric breaking. The various vertical structures of these cyclonic or anticyclonic vortices are not enough to account for the observed differences in MW cyclone and anticyclone stability properties. As far as oceanic vortex stability is concerned, our study is still idealized. Other effects like frontality (large isopycnal deviations) or nonlinearity (Rossby numbers of order unity), will have to be considered in a multi-layer shallow-water model or in a primitive equation model with continuous stratification. External influences on vortex stability, such as that of waves, of surrounding currents or of bottom topography (Sokolovskiy et al 2013), will also have to be studied. Our study is only a first step in that direction.

\section{Acknowledgements}

The authors dedicate this paper to the memory of their colleague Dr Bach Lien Hua, who provided suggestions at the early stage of this work. The first (second) author acknowledges the support of CNRS (RFBR) under the Russia-France PICS program "Geophysical Vortices". Thanks are due to the two anonymous referees for their indepth analysis and fine suggestions. The authors also thank Pr Yasuhide Fukumoto for his help with this paper.

\section{Appendix: Complements on linear instability}

This short complement presents the linear instability of vortices with different radial profiles of velocity to assess the influence of these profiles on our results. Firstly, we consider the velocity profile

$$
V_{j}=U_{j} \frac{R}{r}\left[\exp \left(-r^{2} / R^{2}\right)-1\right]
$$

which corresponds to a Gaussian mean relative vorticity

$$
\nabla^{2} \Psi_{j}=-2 U_{j} / R \exp \left(-r^{2} / R^{2}\right)
$$

This relative vorticity profile is known to be barotropically stable in two-dimensional flows (see again Carton and McWilliams (1989)). Here it can be sensitive to both barotropic and baroclinic instabilities. Indeed, the radial gradient of potential vorticity for this vortex changes sign mostly along the vertical (as for the reference case vortices), but also horizontally. The growth rates for mode $l=2$ for cyclones and anticyclones with velocity profile (equation (9)), are drawn on figure 13. Clearly, there are few differences in linear instability between our reference case and these vortices, in sensitivity to the layerwise velocity ratios. These vortices are slightly more unstable than those of the reference case.

Finally, we present another velocity profile which again grows linearly in the core and decays exponentially in the periphery of the vortex (note that this is another 

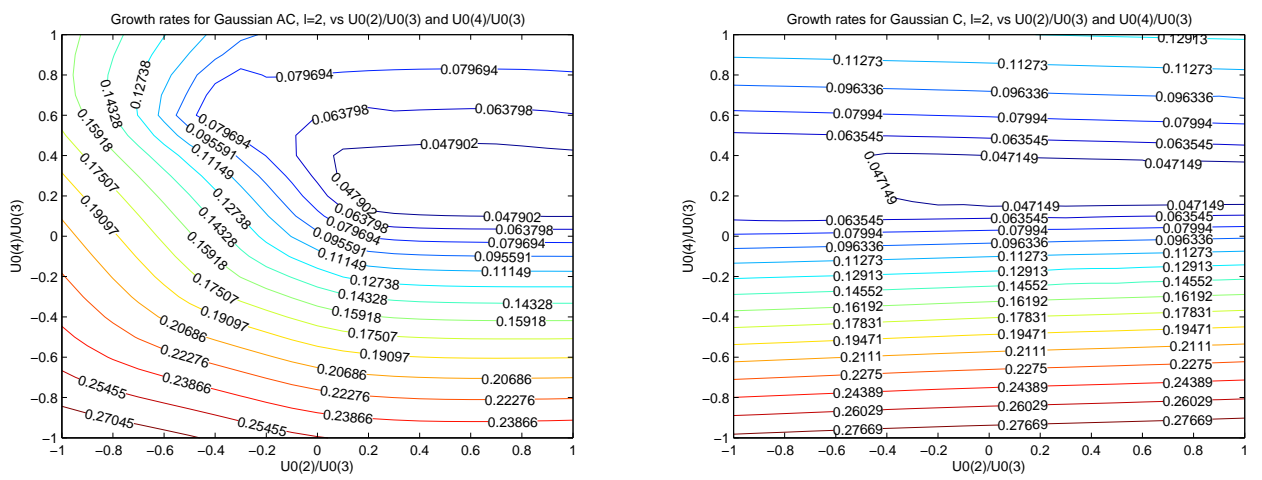

Figure 13. Isolines of normalized growth rates of elliptical normal modes, in the $U_{1} / U_{2}, U_{3} / U_{2}$ plane for Gaussian anticyclones (left), and in the $U_{2} / U_{3}, U_{4} / U_{3}$ plane for Gaussian cyclones (right).
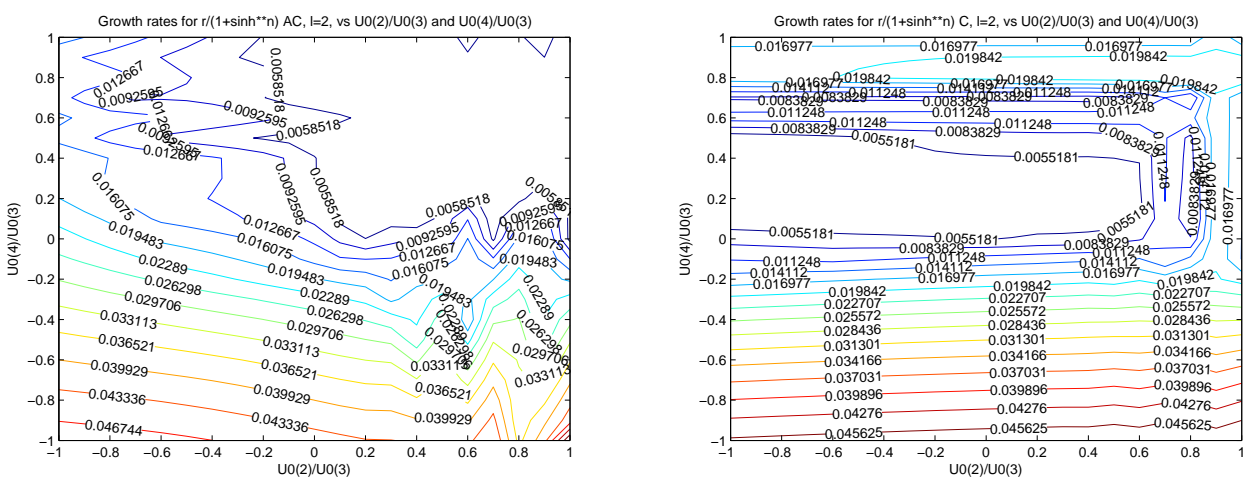

Figure 14. Isolines of normalized growth rates of elliptical normal modes, in the $U_{1} / U_{2}, U_{3} / U_{2}$ plane for $r /\left(1+\sinh ^{2}(r)\right)$ anticyclones (left), and in the $U_{2} / U_{3}, U_{4} / U_{3}$ plane for the cyclones (right).

possibility for meddy velocity profiles)

$$
V_{j}=U_{j} \frac{r / R}{1+\sinh ^{2}(r / R)} .
$$

Figure 14 presents the growth rates for cyclones and anticyclones with this profile (equation (11)), which is less steep than our reference profile (equation (4) with $\alpha=2$ ). The patterns for the instability of cyclones and anticyclones are again globally comparable to those of the reference case, but with much smaller growth rates. It is of interest to consider also more compact vortices, with velocity profiles varying as $(r / R) /\left(1+\sinh ^{n}(r / R)\right.$ where $n$ is large. Then, the external velocity profile goes as $r \exp (-n r / R)$; this means that the apparent size of the vortices is smaller (in $R / n)$, and since baroclinic instability prevails, the vortices become less unstable as $n$ increase. For $n=4$, the vortices are linearly stable. 


\section{References}

Ambar I, Serra N, Neves F and Ferreira T 2008 Observations of the Mediterranean Undercurrent and eddies in the Gulf of Cadiz during 2001 J. Marine Syst. 71 195-220 Barbosa Aguiar A, Peliz A and Carton X 2013 A census of meddies in a long-term, high-resolution simulation Prog. Oceanogr. 116 80-94

Carton X and McWilliams J C 1989 Barotropic and baroclinic instabilities of axisymmetric vortices in a QG model Mesoscale/Synoptic Coherent Structures in Geophysical Turbulence (Elsevier Oceanographic Series vol 50) ed J C J Nihoul and B M Jamart (Amsterdam: Elsevier) pp.225-244

Carton X, Chérubin L, Paillet J, Morel Y, Serpette A and Le Cann B 2002 Meddy coupling with a deep cyclone in the Gulf of Cadiz J. Marine Syst. 32 13-42

Carton X, Daniault N, Alves J, Chérubin L and Ambar I 2010 Meddy dynamics and interaction with neighboring eddies southwest of Portugal : observations and modeling J. Geophys. Res. 115 C06017

Chérubin L, Carton X, Paillet J, Morel Y and Serpette A 2000 Instability of the Mediterranean Water undercurrents southwest of Portugal: effects of baroclinicity and of topography Oceanol. Acta 23 551-573

Chérubin L, Carton X and Dritschel D G 2007 Vortex dipole formation by baroclinic instability of boundary currents J. Phys. Oceanogr. 37 1661-1677

Madelain F 1970 Influence de la topographie de fond sur l'écoulement méditerranéen entre le détroit de Gibraltar et le cap Saint Vincent Cah. Océanogr. 22 43-61

Ménesguen C, Hua B L, Carton X, Klingelhöfer F and Schnürle P 2012 Arms winding around a Meddy seen in seismic reflection data south of the Azores front Geophys. Res.

Lett. 39 L05604

NGuyen H Y, Hua B L, Schopp R and Carton X 2012 Slow quasi-geostrophic unstable modes of a lens-like vortex in a continuously stratified flow Geophys. Astrophys. Fluid Dyn., 106 305-319

Paillet J, Le Cann B, Carton X, Morel Y and Serpette A 2002 Dynamics and evolution of a northern meddy J. Phys. Oceanogr. 32 55-79

Serra N and Ambar I 2002 Eddy generation in the Mediterranean undercurrent Deep Sea Res. 49 4225-4243

Sokolovskiy M A, Filyushkin B N and Carton X 2013 Dynamics of intrathermocline vortices in a gyre flow over a seamount chain Ocean Dyn. 63 741-760

Tychensky A and Carton X 1998 Hydrological and dynamical characterization of Meddies in the Azores region: A paradigm for baroclinic vortex dynamics J. Geophys. Res. 103 25061-25079 